\title{
CÁLCULO COMPUTACIONAL DOS MODOS E DAS FREQUENCIAS NATURAIS DE VIBRAÇÃO DE UMA VIGA BIENGASTADA PELO MÉTODO DOS MODOS ASSUMIDOS
}

Gustavo Figueiredo Formágio; Daniele Araujo Altran; Filipe Bittencourt Figueiredo; Cassio Fabian

Sarquis De Campos

Universidade do Oeste Paulista - UNOESTE, Curso de Engenharia Civil, Presidente Prudente, SP. E-mail: cassio@unoeste.br.

\begin{abstract}
RESUMO
O estudo da dinâmica de estruturas sejam elas rígidas ou flexíveis é de grande importância para a engenharia civil como método de prevenção de possíveis problemas estruturais, tais como trincas, deslocamentos, rupturas parciais ou totais estimulados por processos vibracionais. Este trabalho teve como objetivo determinar computacionalmente os quatro primeiros modos de vibração e suas respectivas frequências naturais para uma viga biengastada empregando o método dos modos assumidos. A parte flexível da viga foi modelada de modo que sua flexibilidade corresponde à uma função com variação espacial neste método. As frequências de vibração ou movimentos flexíveis naturais da estrutura foram obtidos bem como as quatro representações gráficas dos primeiros modos de vibração. As respectivas frequências obtidas foram $7,90 \mathrm{~Hz}, 13,12$ $\mathrm{Hz}, 18,38 \mathrm{~Hz}$ e $23,63 \mathrm{~Hz}$ aproximadamente e respectivamente. Conclui-se que a partir do método proposto, utilizando softwares de programação aberta e especificações técnicas de uma viga pode-se obter suas frequências naturais de vibração.
\end{abstract}

Palavras-chave: frequências de vibração, estruturas, viga biengastada

\section{COMPUTATIONAL CALCULATION OF NATURAL FREQUENCIES AND MODES OF VIBRATION OF A BEAM BY METHOD BIENGASTADA OF ASSUMED}

\begin{abstract}
The study of the dynamics of structures whether rigid or flexible is of great importance to the civil engineering as a method of prevention of possible structural problems, such as broken, offsets, partial or full breaks stimulated by vibrational process. This work had as objective to determine computationally the first four vibration modes and their respective natural frequencies for a beam using the biengastada method of the given modes. The soft portion of the beam was modeled so that your flexibility corresponds to a function with spatial variation in this method. The frequencies of vibration or flexible structure's natural movements were obtained as well as the four graphic representations of the first vibration modes. The respective frequencies obtained were $7.90 \mathrm{~Hz}, 13.12 \mathrm{~Hz}, 18.38 \mathrm{~Hz}$ and $23.63 \mathrm{~Hz}$ about and respectively. It is concluded that from the proposed method, using open programming software and technical specifications of a beam can obtain their natural frequencies of vibration.
\end{abstract}

Keywords: frequencies of vibration, structures, beam clamped-clamped. 


\section{INTRODUÇÃO}

A Engenharia Estrutural área da Engenharia Civil é de suma importância para a otimização e dimensionamento de projetos, pois geralmente as estruturas são fabricadas por meio da técnica de elementos estruturais, através das quais, as cargas solicitantes são transmitidas do ápice das estruturas até as suas fundações. Dentre os elementos estruturais amplamente empregados, temos as lajes, as vigas e os pilares.

Neste contexto, a análise estrutural dinâmica tem por finalidade quantificar os esforços internos, as velocidades, as acelerações e os deslocamentos que são desenvolvidos em qualquer sistema estrutural, quando o mesmo é submetido a um carregamento arbitrário, cuja intensidade, direção e sentido variem com o tempo. (RODRIGUES, 1997)

$\mathrm{Na}$ dinâmica das estruturas, uma grande preocupação, é ocorrência de vibrações excessivas, indesejáveis do ponto de vista estrutural, já que podem causar danos na estrutura devido ao alto valor dos esforços solicitantes provocados. As vibrações também podem ocasionar a fadiga dos elementos estruturais, além de provocar desconforto nos indivíduos que utilizam a estrutura regularmente, além de, comprometer sua segurança. (LARA, 2007).

Desse modo, desde a disseminação da informática ocorrida no final da década de 1980, são promovidos muitos avanços tecnológicos, com grande aumento da capacidade de armazenamento, gerenciamento e processamento de dados apresentados pelos computadores de pequeno porte, o Engenheiro de Estruturas passou a ter acesso a equipamentos e programas computacionais que possibilitam uma análise estrutural baseada em modelos mais refinados, proporcionando um aumento da confiabilidade e diminuição de custos dos projetos e das construções. (WAIDEMAN, 2004).

Neste cenário, no estudo da dinâmica das estruturas, as ferramentas computacionais, baseadas em métodos numéricos, são de grande importância, pois, auxiliam os profissionais a obterem, com maior agilidade, projetos de alta qualidade, diminuindo custos e prazos.

Normalmente, a utilização de modelos numéricos está associada à concepção e projeto de novas estruturas ou então a atividades relacionadas com o acompanhamento e/ou a avaliação de segurança de estruturas existentes que apresentem um risco potencial significativo. (MENDES E OLIVEIRA, 2008).

A pesquisa da dinâmica de estruturas, tanto estas sendo rígidas ou flexíveis sofrendo a ação de forças externas, como ventos, ondas marítimas regulares, vibrações mecânicas de qualquer ordem, sendo de fundamental importância para mensurar e prevenir de possíveis problemas estruturais, tais como trincas, deslocamentos, rupturas parciais ou totais dessas estruturas.

O estudo de vigas é de essencial importância, sabendo da sua relevante função estrutural. A análise das respostas estáticas e dinâmicas dos componentes estruturais, tais como as vigas, sob a ação de várias condições de carga, é muito útil para a modelagem e análise do comportamento de estruturas mais reais e complexas sujeitas a carregamentos similares.

Para tanto, faz-se necessário conhecer as frequências e os modos naturais de vibração da estrutura.

Este trabalho dá continuidade nas atividades de pesquisas na área de dinâmica estrutural e modelagem computacional de sistemas dinâmicos da Faculdade de Engenharia "Conselheiro Algacyr Munhoz Maeder" de Presidente Prudente-SP, iniciada em 2014 com o trabalho "Análise Computacional Dos Modos E Frequências Naturais De Vibração De Uma Viga Engastada-Livre" de Campos, et al.

Objetivou-se aqui implementar computacionalmente um programa para calcular as quatro primeiras frequências naturais de vibração de uma viga biengastada utilizando o método dos modos assumidos, bem como suas respectivas representações gráficas. 


\section{METODOLOGIA}

Na figura 1 apresenta-se o modelo de uma viga biengastada indeformada empregada neste trabalho e o deslocamento flexível da viga em função do comprimento e tempo quando deformada.

Figura 1. Deformação em uma viga biengastada

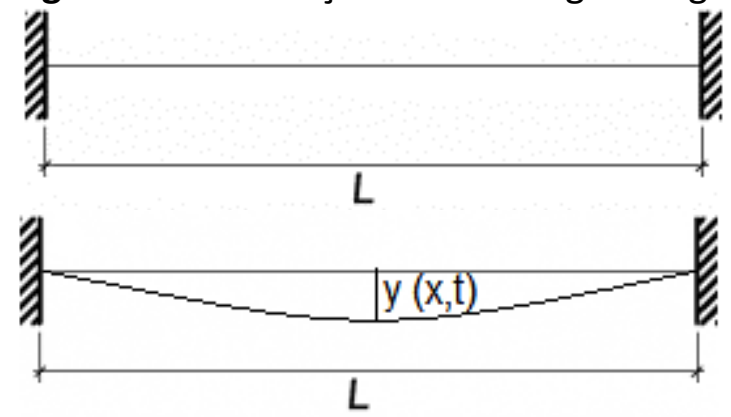

Fonte: (Autoria própria).

Conforme Junkins (1993) mostra, o meio usado para modelar a flexibilidade da estrutura no movimento da viga consiste no método conhecido como método dos modos assumidos, onde a solução associada as vibrações do sistema pode ser escrita na forma:

$\mathrm{y}(\mathrm{x}, \mathrm{t})=\sum_{\mathrm{i}=1}^{\mathrm{n}} \Phi_{i}(\mathrm{x}) q_{i}(t)$

onde,

$y(x, t)$ - corresponde à deformação da viga em função do comprimento $(m)$ e tempo $(s)$;

$\Phi_{\mathrm{i}}(\mathrm{x})$ - denota as funções de forma escolhidas para descrever as deflexões das vigas ao longo de seu comprimento;

$q_{i}(t)$ - indica as coordenadas generalizadas relacionadas aos deslocamentos flexíveis dependentes do tempo.

Neste trabalho usou-se apenas a função $\Phi_{i}(x)$ como suficientes e necessárias para se obter as formas dos quatro primeiros modos de vibração, não levando em consideração a variação temporal. (MEIROVITCH, 1967)

A função de forma, assumida para viga biengastada foi extraída do trabalho de Young e Felgar (1949), representando os deslocamentos flexíveis do sistema.

$$
\Phi_{i}(x)=\cosh \left(\beta_{i} \cdot x\right)-\cos \left(\beta_{i} \cdot x\right)-\alpha_{n} \cdot\left[\operatorname{senh}\left(\beta_{i} \cdot x\right)-\operatorname{sen}\left(\beta_{i} \cdot x\right)\right]
$$

Com $x$ correspondendo ao comprimento da viga considerada de 0 à $L, \beta$ constituindo constantes relacionadas às frequências naturais de vibrações e $\alpha_{n}$ valores característicos do tipo de viga considerada no sistema, no caso aqui, biengastada. Os valores de $\alpha$ e $\beta$ foram extraídos de "Tables of Characteristic Functions Representing Normal Modes of Vibration of a Beam" publicado por Dana Young e Robert, P. Felgar Jr (1949) e estão apresentados na tabela 1. 
Tabela 1. Valores de $\alpha$ e e $\beta$.

\begin{tabular}{|c|c|c|}
\hline Modo (n) & $\boldsymbol{\beta}$ & $\boldsymbol{\alpha}_{\mathbf{n}}$ \\
\hline $\mathbf{1 0}$ & 4,7300408 & 0,9825022158 \\
\hline $\mathbf{2 o}$ & 7,8532046 & 1,000777311 \\
\hline $\mathbf{3 o}$ & 10,9956058 & 0,9999664501 \\
\hline $\mathbf{4}$ o & 14,1371655 & 1,000001450 \\
\hline
\end{tabular}

Fonte: (Young e Felgar, 1949).

As condições de contorno para vigas biengastadas são de que o deslocamento $\emptyset_{i}(x)=0 \mathrm{e}$ $\emptyset_{i}(L)=0$ garantindo que não haja vibração nestes pontos e a função apresentada anteriormente satisfaz estas condições de contorno geométricas. (THOMSON, 1981)

Terminado a modelagem teórica da viga e encontrada a função de forma condizente ao modelo, deu-se início à implementação computacional através dos softwares Matlab ${ }^{\circledR}$ e Maple ${ }^{\circledR}$.

\section{RESULTADOS}

Para modelagem computacional escolheu-se uma viga de concreto com seção quadrada possuindo as seguintes especificações.

Tabela 2. Especificações da viga adotada.

\begin{tabular}{|l|l|l|}
\hline Massa específica & $\rho$ & $2400 \mathrm{Kg} / \mathrm{m}^{3}$ \\
\hline Massa da viga & $m$ & $96 \mathrm{Kg}$ \\
\hline Área de seção & $A$ & $0,04 \mathrm{~m}^{2}$ \\
\hline Comprimento & $L$ & $1,0 \mathrm{~m}$ \\
\hline Módulo de Elasticidade & $E$ & $30641,97 \mathrm{MPa}$ \\
\hline Momento de Inércia & $I$ & $8,64.10^{-6} \mathrm{~m}^{4}$ \\
\hline
\end{tabular}

A partir dos softwares implementou-se a equação 1 com os dados das tabelas 1 e 2 pôdese obter os quatro primeiros modos de vibração da viga biengastada sendo estes representados nos gráficos seguintes. 
Figura 2. Representação Gráfica dos modos de vibração.

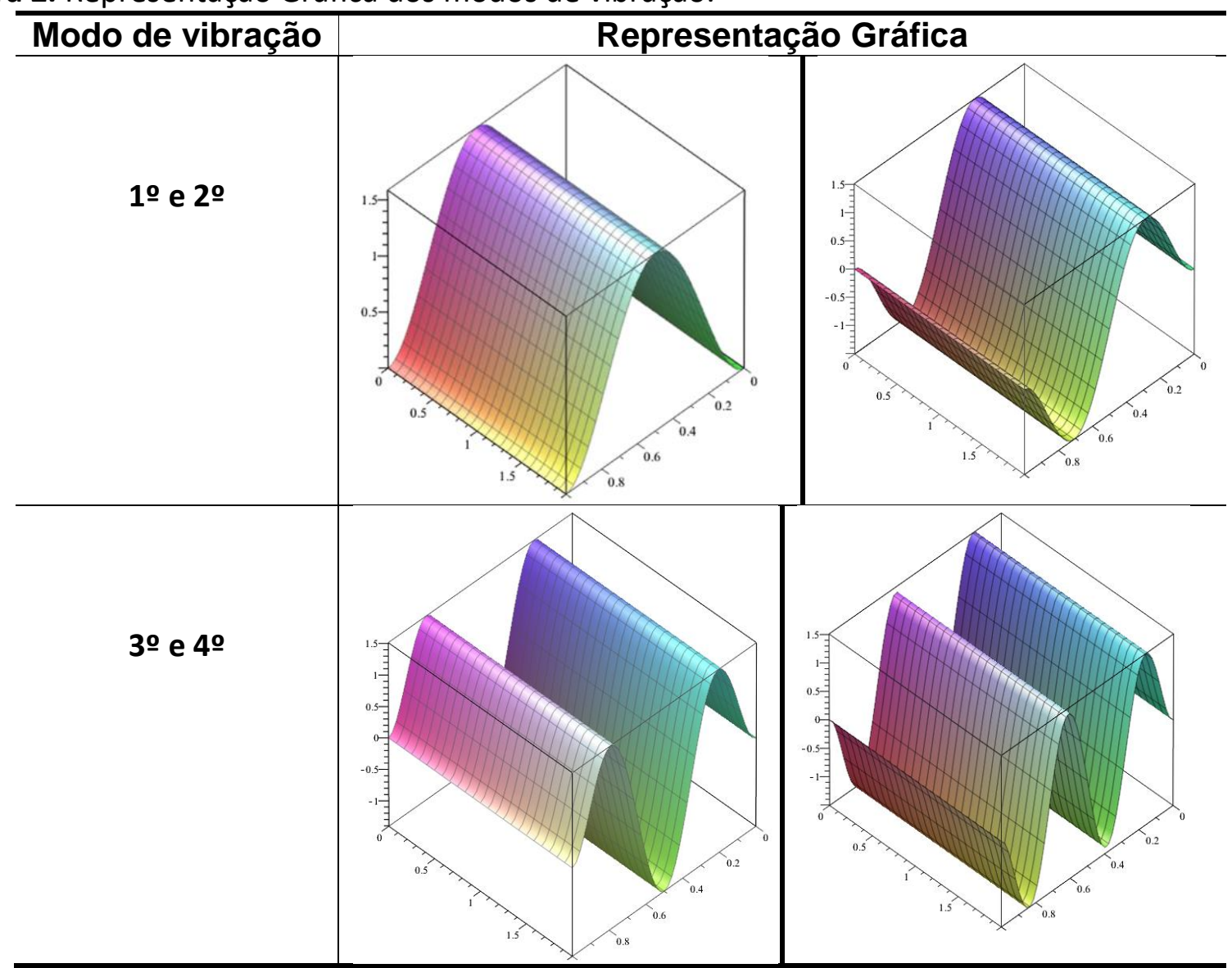

Pode-se observar pela figura 2 os quatro primeiros modos de vibração da viga biengastada onde as respectivas frequências de vibração podem ser calculadas segundo Young e Felgar (1949), através da:

$$
\omega_{i}=\beta_{i} \cdot \sqrt{\frac{E . I}{\rho}}
$$

onde, $i$ representa o $i$-ésimo modo de vibração.

Desta forma, utilizando os valores disponíveis nas tabelas 1 e 2, as frequências para os quatro primeiros modos de vibração da viga proposta são:

Tabela 3. Frequências naturais de Vibração

\begin{tabular}{|c|c|c|}
\hline Modo & Frequências naturais (rad/s) & Frequências Naturais (Hz) \\
\hline $\mathbf{1}$ & 49,6791455 & 7,9066815 \\
\hline $\mathbf{2}$ o & 82,4814225 & 13,1273261 \\
\hline $\mathbf{3} \boldsymbol{0}$ & 115,4857482 & 18,3801276 \\
\hline $\mathbf{4 0}$ & 148,4812355 & 23,6315225 \\
\hline
\end{tabular}

A tabela 3 apresenta as frequências naturais de vibração em rad/s e Hz (Hertz) para a viga biengastada proposta. 
Levando-se em consideração massa específica, módulo de elasticidade, momento de inércia e consequentemente a geometria da viga, observa-se que as frequências são únicas para cada tipo de material e condições de contorno.

\section{CONCLUSÃO}

Neste artigo foi apresentado os modos de vibração de uma viga biengastada e suas respectivas frequências naturais de acordo com o método dos modos assumidos.

Especificamente foi possível se obter as representações gráficas dos quatro primeiros modos de vibração bem como suas respectivas frequências, como sendo de $7,90 \mathrm{~Hz}, 13,12 \mathrm{~Hz}, 18,38 \mathrm{~Hz}$ e $23,63 \mathrm{~Hz}$, aproximada e respectivamente

Conclui-se que a partir do método proposto, utilizando softwares de programação aberta e especificações técnicas de uma viga pode-se obter suas frequências naturais de vibração.

Espera-se que o modelo matemático e computacional desenvolvido seja empregado em novas atividades de pesquisa e de campo na área de engenharia civil nesta instituição e que ainda sirva de base para novos estudos de estruturas mais complexas.

Sugere-se que a mesma modelagem seja realizada utilizando softwares específicos a fim de se comprovar por comparação que é possível modelar estruturas dinâmicas a partir de softwares matemáticos de programação livre e ainda novos cálculos com novos tipos de vínculos.

\section{REFERÊNCIAS}

CAMPOS, C. F. S. de; Et al. Análise Computacional Dos Modos E Frequências Naturais De Vibração De Uma Viga Engastada-Livre. Colloquium Exactarum, v. 6, n.1, Jan-Jun. 2014, p.114 - 127, https://doi.org/10.5747/ce.2014.v06.n1.e072

LARA V. Controle de Vibrações em Vigas Metálicas com Distintas Configurações de Apoio Utilizando Amortecedores de Massa Sintonizados. Dissertação (Mestrado). Departamento de Engenharia Civil e Ambiental. Universidade de Brasília. Brasília. 2007.

JUNKINS, J. L.; KIM, Y. Introduction to Dynamics and Control of Flexible Structures. Washington: American Institute of Aeronautics and Astronautics. 1993, https://doi.org/10.2514/4.862076.

MEIROVITCH, L. Analytical Methods in Vibrations. London: The Macmillan Company. 1967.

MENDES, P.; OLIVEIRA, S. Análise Dinâmica de Estruturas: Utilização Integrada de Modelos de Identificação Modal e Modelos de Elementos Finitos. Laboratório Nacional de Engenharia Civil. Lisboa. 2008.

RODRIGUES, R. O. Análise Dinâmica Bi-Dimensional Não-Linear Física e Geométrica de Treliças de Aço e Pórticos de Concreto Armado. Tese (Doutorado). Escola de Engenharia de São Carlos Universidade de São Paulo. São Carlos. 1997.

THOMSON, W. T. Theory of Vibration with Applications. New Jersey: Prentice Hall. 1981.

WAIDEMAN, L. Análise dinâmica de placas delgadas utilizando elementos finitos triangulares e retangulares. Dissertação (Mestrado) - Faculdade de Engenharia, Universidade Estadual Paulista, Ilha Solteira. 2004.

YOUNG, D.; FELGAR, P. Jr. Tables of Characteristic Functions Representing Normal Modes of Vibration of a Beam. The University of Texas. Texas. 1949. 